\title{
Les rencontres entre médiateurs de santé pairs et usagers de la psychiatrie en France : caractéristiques générales et effets du dispositif sur les représentations des usagers. Partie 1 Encounters between peer workers and users of psychiatry in France: general characteristics and effects of the device on the representations of users. Part 1
}

\author{
Lise Demailly et Nadia Garnoussi
}

Volume 40, numéro 1, printemps 2015

Partenariats patients en santé mentale

URI : https://id.erudit.org/iderudit/1032389ar

DOI : https://doi.org/10.7202/1032389ar

\section{Aller au sommaire du numéro}

\section{Éditeur(s)}

Revue Santé mentale au Québec

ISSN

0383-6320 (imprimé)

1708-3923 (numérique)

Découvrir la revue

Citer cet article

Demailly, L. \& Garnoussi, N. (2015). Les rencontres entre médiateurs de santé pairs et usagers de la psychiatrie en France : caractéristiques générales et effets du dispositif sur les représentations des usagers. Partie 1. Santé mentale au Québec, 40(1), 171-187. https://doi.org/10.7202/1032389ar

\section{Résumé de l'article}

Objectif : Cet article traite des rencontres entre des usagers de la psychiatrie et de nouveaux professionnels, les médiateurs de santé pairs (MSP), formés dans le cadre d'un programme expérimental français dirigé par le CCOMS (Centre collaborateur de l'OMS). Les données empiriques que nous présentons ici sont issues d'une enquête sociologique qualitative qui a participé à l'évaluation de ce programme, menée entre 2012 et 2014. Le programme MSP consiste à embaucher dans des services de psychiatrie publique, au terme d'une formation de huit semaines équivalant à un diplôme universitaire et d'une année de stage, d'(ex-)usagers de la psychiatrie, des personnes « ayant eu ou ayant encore des troubles psychiques, rétablis ou en voie de rétablissement ». Au-delà de la création d'une nouvelle profession dans le champ de la santé mentale, le programme s'est donné pour objectif la transformation des pratiques et des représentations des équipes de psychiatrie publique.

Méthode : Soutenu par trois Agences Régionales de Santé (ARS), il a débuté avec 29 candidats à des postes de MSP et visait après la phase expérimentale à être étendu à d'autres régions, voire généralisé. À l'heure actuelle, 15 MSP sont en activité, sur contrat à durée déterminée renouvelable de la catégorie $\mathrm{C}$ de la fonction publique. Nous nous intéressons plus spécifiquement ici, dans une démarche ethnographique, aux rencontres entre patients et MSP : à leur contenu, leur forme, leur style, aux caractéristiques de ces interactions ainsi qu'à certains de leurs effets. Le matériel d'enquête exploité est composé de 74 entretiens semi-directifs avec les usagers et d'observations conduites dans les services, ayant permis d'analyser des échanges à la fois formels et informels.

Résultats : Nous avons organisé l'analyse autour de quatre grands thèmes. Les deux premiers, qui font l'objet d'un premier article, concernent les caractéristiques générales de la rencontre et les effets du dispositif sur les représentations des usagers quant à la maladie et au système de soins. Les déterminants communs des rencontres que nous avons pu dégager sont les suivants : leur caractère volontaire ; une normativité moindre sur les comportements ; une offre de temps et de disponibilité ; un rapport distant à la médicamentation ; un déclivage vie privée/vie professionnelle ; une gestion spécifique de la distance et de la familiarité. Concernant les représentations de la maladie et du soin, nous montrons que les MSP contribuent à diffuser activement une idéologie du rétablissement fondée sur une conception positiviste de la personnalité. Ils s'autorisent également une certaine critique des autres professionnels et du système de soins et cherchent à s'en distinguer en privilégiant une philosophie de la patience et du " petit pas ».
Tous droits réservés @ Département de psychiatrie de l’Université de Montréal, 2015
Cest protégé par la loi sur le droit d'auteur. L’utilisation des services d'Érudit (y compris la reproduction) est assujettie à sa politique d'utilisation que vous pouvez consulter en ligne.

https://apropos.erudit.org/fr/usagers/politique-dutilisation/ 


\title{
Les rencontres entre médiateurs de santé pairs et usagers de la psychiatrie en France: caractéristiques générales et effets du dispositif sur les représentations des usagers PARTIE 1
}

\author{
Lise Demailly
}

Nadia Garnoussi ${ }^{\mathrm{b}}$

RÉSUMÉ Objectif: Cet article traite des rencontres entre des usagers de la psychiatrie et de nouveaux professionnels, les médiateurs de santé pairs (MSP), formés dans le cadre d'un programme expérimental français dirigé par le CCOMS (Centre collaborateur de l'OMS). Les données empiriques que nous présentons ici sont issues d'une enquête sociologique qualitative qui a participé à l'évaluation de ce programme, menée entre 2012 et 2014 . Le programme MSP consiste à embaucher dans des services de psychiatrie publique, au terme d'une formation de huit semaines équivalant à un diplôme universitaire et d'une année de stage, d'(ex-) usagers de la psychiatrie, des personnes «ayant eu ou ayant encore des troubles psychiques, rétablis ou en voie de rétablissement». Au-delà de la création d'une nouvelle profession dans le champ de la santé mentale, le programme s'est donné pour objectif la transformation des pratiques et des représentations des équipes de psychiatrie publique.

Méthode: Soutenu par trois Agences Régionales de Santé (ARS), il a débuté avec 29 candidats à des postes de MSP et visait après la phase expérimentale à être

a. Professeure émérite de sociologie, Université de Lille, CLERSE, MESHS-CNRS.

b. Maître de conférence en sociologie, Université de Lille III, Centre de Recherche Individus, Épreuves, Sociétés (CeRIES). 
étendu à d'autres régions, voire généralisé. À l'heure actuelle, 15 MSP sont en activité, sur contrat à durée déterminée renouvelable de la catégorie $\mathrm{C}$ de la fonction publique. Nous nous intéressons plus spécifiquement ici, dans une démarche ethnographique, aux rencontres entre patients et MSP: à leur contenu, leur forme, leur style, aux caractéristiques de ces interactions ainsi qu'à certains de leurs effets. Le matériel d'enquête exploité est composé de 74 entretiens semi-directifs avec les usagers et d'observations conduites dans les services, ayant permis d'analyser des échanges à la fois formels et informels.

Résultats: Nous avons organisé l'analyse autour de quatre grands thèmes. Les deux premiers, qui font l'objet d'un premier article, concernent les caractéristiques générales de la rencontre et les effets du dispositif sur les représentations des usagers quant à la maladie et au système de soins. Les déterminants communs des rencontres que nous avons pu dégager sont les suivants: leur caractère volontaire; une normativité moindre sur les comportements; une offre de temps et de disponibilité; un rapport distant à la médicamentation; un déclivage vie privée/vie professionnelle; une gestion spécifique de la distance et de la familiarité. Concernant les représentations de la maladie et du soin, nous montrons que les MSP contribuent à diffuser activement une idéologie du rétablissement fondée sur une conception positiviste de la personnalité. Ils s'autorisent également une certaine critique des autres professionnels et du système de soins et cherchent à s'en distinguer en privilégiant une philosophie de la patience et du «petit pas».

MoTS CLÉS médiateurs de santé pairs, savoir expérientiel, santé mentale, rencontres MSP/usagers

\section{Encounters between peer workers and users of psychiatry in France: general characteristics and effects of the device on the representations of users. PART 1}

ABSTRACT Objective: This series of two parent papers describes, as part of an experimental program in France, the meetings that took place between consumers of psychiatric services and peer mentors. We report on the shape, style and characteristics of these interactions. Some of their effects are also reported. The peer mentors are hospital employees who are hired through renewable contracts or, for some, on permanent contracts. The program was led by the World Health Organization Collaborating Centre for Mental Health for France, with the intention, beyond the creation of a "new profession," of transforming current practices and representations of public psychiatry teams towards recovery. The first paper discusses the general characteristics of the consumer/peer mentor meetings and the effects of this mechanism on the representations of the consumers about mental illness and more broadly about the health care system. The second paper reports on the therapeutic effects of these meetings. Some limitations and pitfalls are highlighted. 
Methods: The program under study involved the hiring of (ex-) consumers of psychiatric services $(n=29)$. These peer mentors are individuals "who have had, or still have psychiatric disorders, and have experienced a process of recovery." They were deployed in public psychiatric services after having been trainees for a year and having pursued a training of eight weeks, in parallel, that led to a peer mentor university degree. Our sample is mainly composed of patients who met at least once with a peer mentor, and who were recruited during the field survey in the sites (in-hospital, outpatient or in urban settings). We approached and spoke to patients who were likely to agree to be interviewed to share their experience and thoughts. They had to sign consent forms and thus they were told that we were working on this experimental program on peer mentors with a qualitative and inductive approach. The empirical data that is reported here comes from a qualitative sociological investigation that accompanied the evaluation of the implementation of the program between 2012 and 2014. We have conducted 74 interviews with consumers. We have a significant number of observations of the meetings between peer mentors and consumers, some of which were transcribed verbatim. We were also informed by informal discussions with consumers in every service, and through direct interviews with peer mentors, as well as through in situ observations.

Results: We used standardized prompt questions such as: "Did you meet $X$," or "Do you know X?" We had to introduce the terms "peer" or "former patient." In effect, in interviews with study participants it appeared that this " $X$ " was sometimes perceived as more of a nurse, and that they were not aware that this person was a "peer mentor" or a former patient. The insistent style of some questions and the context of what could appear to be a formal assessment of a person's work might explain why study participants seemed inclined to give positive comments. A bias was also possible when study participants were recruited and "prepared" by the treatment team for the interview. Despite these limitations, we can highlight the quality of the data.

Conclusion: By the end of the experiment, 15 peer mentors were able to find and make their place in various services, despite important differences between situations with regards to their position in the institution, their degree of autonomy and responsibility, their working arrangements with other professionals, and their actual work. However, we were able to identify common determinants: the voluntary nature of the meetings; less normativity on behavior; the supply of time and availability; a distant relation to medical drugs; a blurring of boundaries between private and professional life; specific management of distance and familiarity. Concerning representations of illness and care, we found that the peer mentors actively contribute to disseminate a vision of recovery based on a positivist conception of personality. They also allow some criticism of other professionals and of the healthcare system. Consumers, in turn, evoke interactions based on the ease of contact, proximity and availability of peer mentors. This proximity can be linked to 
a form of camaraderie in the institutional context of care but can also lead consumers to see, in the peer mentor, a figure of "hope."

KEYWORDS peer mentors, experiential knowledge, mental health, meetings peer mentors and consumers of psychiatric services

Ce texte décrit, dans le cadre d'un programme expérimental en France, les rencontres entre patients et Médiateurs de santé pairs en santé mentale: leur forme, leur style, les caractéristiques de ces interactions, certains de leurs effets. Il s'agit d'une étude qualitative et inductive.

Le programme en question (2012-2014) consiste en l'embauche d'(ex-) usagers de la psychiatrie (MSP), autrement dit de personnes «ayant eu ou ayant encore des troubles psychiques, rétablis ou en voie de rétablissement», au nombre initial de 29 , dans des services de psychiatrie publique, après qu'elles ont été stagiaires un an et qu'elles ont suivi parallèlement une formation de huit semaines (un diplôme universitaire de médiateur de santé pair à l'Université de Paris VIII). Les MSP sont actuellement salariés des hôpitaux sur contrat à durée déterminée renouvelable de la catégorie $C$ de la fonction publique ou pour quelques-uns sur contrat à durée indéterminée. Le programme a été dirigé par le Centre collaborateur OMS France pour la santé mentale, qui avait pour objectif, au-delà de la création d'une «nouvelle profession», la transformation des pratiques et des représentations des équipes de psychiatrie publique ${ }^{1}$. Le programme devait en principe, après la phase expérimentale, être étendu par vagues successives à d'autres régions de France.

\section{Méthodologie et objet}

Notre corpus est pour l'essentiel composé de patients qui ont été en contact au moins une fois avec le MSP, et que nous avons recrutés au

1. Demailly, L., Bélart, C., Déchamp Le Roux, C., Dembinski, O., Farnarier, C., Garnoussi, N. et Soulé, J., Le dispositif des médiateurs de santé pairs en santé mentale: une innovation controversée. Rapport final de la recherche évaluative qualitative sur le programme expérimental 2012-2014, Lille, septembre 2014. Convention de recherche Clersé USTL Lille 1/CCOMS EPSM Lille-Métropole et www.ccomssantementalelillefrance.org/ 
fil de l'enquête de terrain dans les sites, en intrahospitalier, ambulatoire ou en ville. D'autres caractéristiques de notre échantillon sont à signaler. Le premier est que nous nous sommes adressées à des patients susceptibles d'accepter de parler. Le second est que nous avons dû faire signer des feuilles de consentement et donc expliquer que nous travaillions à un programme expérimental et aux activités des «médiateurs de santé pairs ». Alors que nous avions prévu un démarrage standardisé d'entretien du type: "avez-vous rencontré $X$, connaissez-vous X?», nous avons été contraintes d'introduire le terme de «médiateur» ou "d'ancien malade». En discussion spontanée en revanche, il arrivait que $\mathrm{X}$ soit perçu(e) comme un(e) infirmier(ère) ou qu'il ne soit pas su que le (la) «médiateur(trice)» était un(e) ex-malade. L'insistance des questions et le cadre de ce qui pouvait apparaître comme une évaluation des personnes poussaient aussi davantage aux appréciations positives ou conformes à ce que les patients pouvaient estimer que l'on attendait d'eux ${ }^{2}$.

Ces réserves faites, nous pouvons souligner la qualité de notre matériel d'enquête. Nous disposons de 74 entretiens avec les usagers (notés «U...»), ce qui constitue environ le tiers des patients suivis par des MSP au premier trimestre 2013. Nous disposons aussi d'un nombre important d'observations de rencontres entre les MSP et les usagers, dont certaines ont donné lieu à un enregistrement (notées «Inter...»), et de discussions informelles avec les usagers, dans tous les services, $y$ compris ceux dans lesquels des entretiens enregistrés n'ont pas eu lieu. Et bien sûr d'entretiens avec les MSP, ainsi que d'observations in situ du travail des MSP (deux jours à trois semaines). Ce premier article présente les caractéristiques générales de la rencontre, ainsi que les effets du dispositif sur les représentations des usagers quant à la maladie en général et au système de soins.

\section{Caractéristiques générales de la rencontre}

La nature et le cadre organisationnel de l'unité de psychiatrie publique dans laquelle travaillent les MSP influencent la forme et le contenu des rencontres: par leurs règles, leurs coutumes, l'autonomie laissée par l'équipe au MSP, par le style des autres professionnels que les patients comparent spontanément au MSP. Le type de patientèle est évidemment très déterminant: les MSP travaillent aussi bien dans des services

2. À noter également un biais quand les usagers étaient choisis et «préparés » aux entretiens par des membres de l'équipe soignante (un cas). 
qui reçoivent des populations extrêmement défavorisées que dans ceux où existe une mixité sociale, dans ceux qui acceptent toute demande et ceux où la relation avec le service s'engage sur la base d'un contrat de soins avec le patient. Les caractéristiques organisationnelles du service et du type d'unité déterminent également le cadre des rencontres (certains MSP rencontrent fréquemment les usagers seuls à seuls, d'autres jamais) ainsi que les activités au cours desquelles elles se vivent: entretiens de face à face en Centre médico-pédagogique ou visite à domicile (VAD); interventions dans un espace collectif comme un hôpital de jour; animation de groupe de parole; etc. La personnalité, l'expérience et les compétences du MSP enfin impriment leur marque à la rencontre. La mobilisation de leur «savoir expérientiel» varie suivant de nombreux paramètres: celui de leur plus ou moins grande connaissance des «pathologies» mais aussi des personnes suivant leur âge, leur classe, leur culture. Pour ces raisons, les rencontres MSP/ patients sont fort singulières. Il y a cependant des éléments communs que l'on retrouve dans toutes les rencontres MSP/patients et qui les spécifient par rapport aux rencontres autres professionnels/patients. C'est cette spécificité globale et ces caractéristiques largement communes que nous voulons dégager.

\section{Caractère volontaire des rencontres}

À la différence de la rencontre avec les autres professionnels, psychiatres et infirmiers, celle d'un MSP est laissée à l'appréciation du patient.

Les soignants, ils sont là, ils distribuent un traitement, toute la journée ils nous prennent en charge d'une certaine façon, d'une manière - c'est obligatoire, tandis qu'avec lui, ce n'est pas obligatoire en fait, et c'est toute la différence (...). (U 76)

Lui il a décidé, je pense, qu'il voulait aider les autres. Ça, c'est déjà louable (...). On peut dire que c'est une bonne personne. Je pense, enfin je ne sais pas, ça se voit qu'il a envie d'aider. Ça, c'est sympa. (U 74)

Normativité moindre quant aux comportements de la vie quotidienne

La normativité paraît aux patients plus faible, notamment par opposition à celle des psychiatres «qui font la morale» (ces propos correspondent à ce que nous avons pu observer en comparant les interactions patients/MSP à des consultations médicales ou des entretiens infirmiers observés dans le cadre de recherches antérieures) et aussi par 
rapport à une majorité d'infirmiers, souvent en position «éducative» par rapport aux patients. Par exemple, sur la propreté des locaux, le ménage, la cigarette, les «bêtises», les MSP sont peu normatifs. Cette moindre normativité peut être due à un savoir de la maladie par l'expérience («Là, j'ai envie de le secouer, mais enfin, je me souviens comme j'étais moi...", dit Roméo), à une moindre intériorisation des préoccupations de santé publique, et plus globalement au décalage entre leur éthos et les normes du monde hospitalier.

S: L'infirmier vous avait fait une réflexion sur le ménage, c'est ça?

U: Tout à fait, oui. Parce que, bon, c'est vrai qu'on peut constater que je ne suis pas très, très ordonnée. Mais, chez moi, c'est mon chez-moi.

S: Bien sûr.

$U$ : Donc, je reçois les gens comme je peux. Et, euh... comme je veux. Et, en fait, il avait fait une réflexion sur la poussière qui avait sur le décodeur qui se trouve là. Et en disant qu'on pouvait carrément écrire son nom dessus, quoi. (Sociologue avec U 12)

Je me sentais pas questionnée ou interrogée comme j'ai pu me sentir parfois par les infirmiers et par les médecins. Pour moi, c'était beaucoup moins intrusif (...). (U 76)

[Faisant allusion à des incidents mettant en jeu une voiture, de la drogue, la police, etc.: ] Enfin, moi, j'avais fait des conneries, Il (le MSP) avait fait des conneries, on en rigolait et y avait plus, pas trop de barrières, quoi. (U 31),

\section{Temps et disponibilité}

Les entretiens ou visites sont plus longs avec un médiateur qu'avec les autres professionnels, parfois plus d'une heure - du moins pour le moment -, le téléphone portable est accessible... L'une des qualités différentielles du médiateur réside dans sa disponibilité et plus généralement dans son accessibilité: la dimension quantitative du temps qu'il peut consacrer aux individus est généralement associée à la facilité d'entrer en relation avec celui-ci, ou encore à l'aisance qu'il autorise dans la communication. «On se sent à l'aise » avec le médiateur remarquent souvent les patients, et participe grandement de cette facilité relationnelle le fait de pouvoir parler «de tout»: les observations confirment que les échanges entre médiateurs et patients sont aussi - et parfois majoritairement - faits de conversations ordinaires.

Le docteur, ça dure 10 minutes-1/4 heure et le médiateur de santé, j'arrive à rester une 1/2 heure à discuter avec lui. Alors que le psy je bavarde un 
peu, enfin c'est technique, je lui parle de la maladie, tout le temps. Alors qu'avec le MSP, je parle de ma vie, ce que j'aime, de ma maladie, mais un peu de tout. Avec le docteur je parle surtout de ce qui m'arrive, mes ennuis quoi. Il y a des petites différences peut-être comme ça (...). Avec le MSP, oui la musique et tout ça, chose que je ne parle pas de trop avec les docteurs. C'est vrai qu'avec le docteur aussi je peux lui expliquer ma musique, mais c'est pas pareil, c'est plus technique avec le docteur qu'avec le médiateur de santé. (Il est) accessible. C'est important. (U 72)

C'est surtout sur la vie quotidienne, surtout ça. Pis des fois on parle aussi de nos goûts - parce que lui il a... je crois qu'il a une formation - il a fait $d u$ dessin beaucoup, et donc des fois, on en parlait, plusieurs fois on en a parlé voilà. (U 75)

Rapport à la médicamentation: les MSP ne «sont pas là pour ça»

La prescription de médicaments est un critère important de distinction entre les différents professionnels que rencontrent les patients. Elle distingue bien sûr en premier lieu la fonction du psychiatre, mais aussi par extension celle des infirmières dont les patients savent qu'elles peuvent influer sur les remaniements du traitement, ses dosages, etc. À deux niveaux, le médiateur peut dès lors affirmer une spécificité. Premièrement, il a lui-même fait l'expérience des psychotropes, élément auquel les patients sont sensibles au nom de la différence entre le discours «théorique» des soignants classiques et l'authenticité du « vécu»: «Lui c'est plus dans le vécu donc c'est plus intéressant » (U 71); «Pour moi, c'est une aide en me disant que quand je parle de quelque chose, il sait ce que je pointe et que des fois [les autres soignants] ils imaginent, ils ont les cours théoriques, mais ils n'ont jamais ressenti physiquement et personnellement» (U 70). Deuxièmement, et c'est un aspect tout aussi déterminant, les échanges portant directement sur les symptômes des troubles ou les effets secondaires des médicaments sont dissociés de la réévaluation dont le traitement peut faire l'objet. Le médiateur dans la plupart des $\operatorname{cas}^{3}$ ne se sent pas tenu de travailler sur l'observance thérapeutique. On retrouve ici chez les patients l'idée que le médiateur, s'il est reconnu comme ayant une «posture professionnelle», n'en devient pas pour autant un professionnel «comme un autre» dans la mesure où, selon eux, il propose une écoute «désintéressée», non orientée par des décisions thérapeutiques à prendre.

3. Au moins jusqu'ici. 
Précisons que cela a été constaté dans les situations où le médiateur n'est pas sous la tutelle du corps soignant, condition à ce que sa fonction soit vraiment distinguée du pouvoir médical.

Ma psy, je ne lui parle plus du tout car je pense qu'elle me chargerait de médicaments, car quand j'étais très envahie, je niais, mais elle me donnait quand même mon traitement car c'est son rôle à jouer. (U 85)

Les médiateurs de santé, comme ils n'ont pas de responsabilités au niveau des médicaments, ils nous prennent tels qu’on est. (U 33)

Les infirmiers sont beaucoup plus dans leur fonction. Il n'y pas de place pour la discussion. Il faut prendre son traitement et c'est point barre. (U 39)

[Le médiateur] il est plutôt dans la conversation. (U 80)

Enfin, il faut dire que les MSP se défendent d'être anti-médicaments, même s'ils évoquent souvent le problème des effets secondaires ainsi que leur influence parfois délétère sur le comportement (par exemple le manque de motivation, l'abattement - certains médiateurs considérant que les soignants sous-estiment parfois ces effets). Mais dans tous les cas, que les médiateurs incitent les patients à parler des traitements ou, au contraire, évitent le sujet, ils reconnaissent quasiment tous que cette question relève in fine de la juridiction médicale. La précaution dont les médiateurs font preuve est motivée en premier lieu par la volonté d'éviter des conflits qui ne manqueraient certainement pas de surgir s'ils se prononçaient «contre» la décision médicale: «Attention, je ne lui dis jamais de ne pas les prendre, je le renvoie au psychiatre.» Ils peuvent toutefois exprimer qu'ils y sont opposés à titre personnel ou que le médicament est nécessaire, mais non suffisant au rétablissement, ou encore encourager le patient à parler au médecin des inconvénients qu'il rencontre.

Moi, je pense qu'on a des forces. Quand on est malade, on croit qu'on n'en a pas, mais on se trompe, c'est la maladie. Mais en réalité, si on persévère, on développe des aptitudes, des schémas de pensée qui nous aident, et qui eux, seront toujours là, sans effets indésirables. (MSP inter E)

Mais s'il faut un médicament pour aller mieux, ben, c'est tout, il faut un médicament. Le souci...

$U:$ Ben ouais, mais quand...

M: ... le souci, c'est qu'on constate...

U: Ça va pas mieux! 
M:... que tu en prends des médicaments et que, ben, ceux que tu prends actuellement, apparemment, c'est pas les bons.

$U$ : Ben ouais. (MSP inter E)

Moindre clivage vie privée/vie professionnelle

L'ampleur de ce dé-clivage, toujours présent, est variable d'un MSP à l'autre, certains ne pratiquant pas l'autodivulgation de leur maladie passée. Dans un cas, le dé-clivage va jusqu'à jouer aussi à propos de la vie «privée » d'un soignant que le MSP a évoqué «naturellement» avec un usager (ce qui fut source de tensions et de débats dans l'équipe). Ce dé-clivage relatif, qui est une technique de travail relationnel, entraîne réciproquement à plus de confidence de la part de l'usager, mais la confidence n'est pas une caractéristique générale et discriminante de la rencontre MSP/patient. S'il y a des MSP auxquels «on dit tout», à d'autres, «on ne dit pas tout». Voici deux situations opposées:

On discute, on peut discuter ouvertement il y a des choses que je n'avais jamais dit à personne même pas à mon psy, je lui ai dit parce qu'on se comprend, notamment au point de vue des médicaments, du conjoint, de la famille, des bêtises que j'ai pu faire par rapport à avant que je sois diagnostiquée [...] elle me comprend [...] elle est passée par là c'est plus facile de parler avec elle qu'avec un psy. (U 4)

Je m'entends très bien avec elle (l'infirmière). Je peux tout lui dire. Parce que bon. Même tout ce qui est relations sexuelles avec mon amie, des choses comme ça, j'arrive à en parler à mon infirmière. Bon, j'en parle pas trop à Dorothée (la MSP), ça la regarde pas. Mais tout ce qui est intime, j'en parle au service médical...

Sociologue: Vous arrivez à en parler à l'infirmière?

U: Ouais. Et à la psychiatre. (U 13)

\section{Familiarité}

Une certaine familiarité, respectueuse, caractérise le contact, spontanée ou utilisée à des fins de facilitation du travail relationnel.

La distance qui s'impose entre les patients et les professionnels, généralement reconnue des deux côtés comme nécessaire, apparaît réduite avec le médiateur. Son attitude, son langage, sa façon d'aborder les patients sont perçus comme contrastant avec ceux des autres professionnels. Cette familiarité est modulée suivant les MSP, car elle est plus ou moins maniée, stratégiquement, à des fins d'entrée en relation. Sur un plan général, les médiateurs sont perçus comme "gentils», «agréables», «souriants»: de fait, et ce contrairement aux autres 
professionnels, ils doivent souvent aller au-devant des patients afin de se faire connaître dans les services et de ne pas dépendre uniquement de la recommandation du médecin. En ce sens, le paraître du médiateur constitue une caractéristique centrale de sa pratique, puisqu'elle est la base d'une construction ultérieure d'une relation plus étroite avec le patient.

Ben, elle est rentrée, elle a dit: "Danièle. » J'ai dit: "Maria. » Et puis, elle m'a tout de suite dit: «Bon, ben, on va se tutoyer, hein! Pour que ce soit plus, plus facile pour travailler.» (U 8)

Déjà, la tenue vestimentaire. Voilà. C'est pas costume, cravate, machin et tout ça. Ce n'est pas tiré à quatre épingles. Il y a la parole aussi qui joue beaucoup. Il y a... Moi, c'est vrai que j'ai horreur qu'on me dise: «Monsieur, vous. » C'est un truc que j'ai horreur. Tout le monde est égal. Moi, j’ai un prénom, c'est Francis. On me dit: «Francis, tu. » Et puis voilà, c'est tout. J'ai pas besoin d'être un monsieur. Je ne suis pas un monsieur, moi. Je suis qu'une personne. (U 42)

La majorité des médiateurs soignent particulièrement la chaleur relationnelle, de telle sorte que leur «sympathie» est parfois ce que les patients retiennent prioritairement de leur présence dans le service.

Quand on lui parle on sent une relation qui se crée, une relation amicale, mais en même temps comme si c'était Noël, quand je dis bonjour, [il répond] «Bonjouuur!!", c'est très jovial, très chaleureux. (U 62)

"[J'ai rencontré le MSP] il y a quelques mois, un petit nombre de mois si je me souviens bien... donc pour l'instant j'ai vraiment rien à lui reprocher, au contraire, si je devais le qualifier par deux adjectifs, je dirais: accessible et sympa. (U 65)

Mais dans d'autres cas, la camaraderie peut être perçue par les patients comme un soutien actif, non superficiel, qui donne à la singularité de la personnalité du médiateur toute sa force.

Tout le monde l'aime bien là-bas, le MSP, c'est un peu le grand frère, quand il vient, il aide pour la montre, pour le téléphone, c'est un médiateur, sa particularité à lui, il aime rigoler, il fait rigoler les autres, mais pour soutenir quoi! Mais c'est quelqu'un il faut qu'il soit là quoi [...] il est dans le pavillon, il fait des scrabbles des échecs, des trucs comme ça, il s'occupe de nous... Le MSP, il peut s'occuper de nous! (U 83) 


\section{Distance spécifique}

Les rencontres ont tendance à se faire au rythme d'une fois par semaine ou une fois tous les quinze jours. Certains MSP ont pu choisir un accompagnement plus soutenu, deux fois par semaine, puis ont fait marche arrière: «Mais on a eu peur, cela devenait trop affectif pour eux et pour moi aussi. » Déroutés par les phénomènes de transfert et de contre-transfert, les MSP les évitent (en partie) et reconstruisent une distance par la baisse de la périodicité des rencontres. Ou bien ils redécouvrent, après avoir beaucoup touché leurs patients, certaines vertus de la distance physique: «Je lui faisais la bise et après, j'ai eu du mal à reprendre une distance» (Roméo).

\section{Influences des MSP sur les représentations qu'ont les usagers de la maladie et du soin}

\section{Rétablissement et chronicité de la maladie}

La présence des MSP contribue à diffuser une certaine idéologie ${ }^{4} \mathrm{du}$ «rétablissement» (recovery) auprès des usagers, auprès de ceux qui sont découragés aussi bien quauprès de ceux qui ont espoir de "guérir». Dans le premier cas, c'est surtout par son exemple personnel que le MSP contribue à accréditer que le rétablissement est possible. Dans le second cas, c'est par les discussions que l'usager apprend qu'il ne doit pas espérer ou viser la guérison, mais le rétablissement, autrement dit qu'il restera probablement malade, mais pourra mener une vie correcte.

On va parler un peu de ce que je faisais et qui j'étais. Donc aujourd'hui, on va prendre un peu de temps pour se connaître, je vais vous expliquer qui je suis, après vous me parlerez un peu de vous. Parce que logiquement, on va se voir, si vous êtes d'accord, une fois tous les quinze jours. (Inter B). MSP

Alors je fais partie d'un nouveau programme qui s'appelle médiateur de santé pair et le but c'est de... au sein des équipes de médecins, d'infirmiers, de mettre des ex-patients. C'est-à-dire que moi et vous, nous sommes pairs, P A I R. Ça veut dire qu'on a des choses en commun. C'est-à-dire que moi je suis un ancien malade, qui a eu des problèmes de santé mentale. J'étais suivi pendant de longues années par une équipe, comme vous pouvez l'être et qui petit à petit s'est rétabli. Donc pour vous,

4. Les partisans du « rétablissement» au sens nord-américain du terme parleraient sans doute de philosophie d'éthique ou d'orientation. Nous l'abordons ici comme un système de représentation. 
c'est surtout le fait de vous dire qu'on peut toujours s'en sortir même si on a un problème de santé mentale, donc là j'ai regardé un petit peu votre dossier. On a plus ou moins la même... Enfin on avait, moi je dis qu'on a en commun, la même maladie. C'est-à-dire que c'est pour vous prouver qu'il y a toujours de l'espoir et que vous pouvez vous en sortir, parce que moi je suis comme vous. J'ai été comme vous et il y a des moments où je suis fragile, où je le suis toujours, mais j'ai réussi petit à petit à reprendre des forces, à bien suivre mon traitement, ça m'arrive encore quand je vais pas bien de prendre...

Parce que nous dans notre maladie on est chronique. Chronique, ça veut dire qu'il y a des moments on ne va pas bien, il y a des moments on va bien... On est toujours comme ça. Donc on a beaucoup de mal parfois à faire des projets, à faire des choses. Est-ce que vous êtes d'accord avec ce que je dis? La chronicité c'est ça, c'est de se dire: «Là je suis bien, mais dans une semaine ou deux je ne serai peut-être pas bien.»Vous voyez, c'est toujours comme ça. Bon moi j'ai cette pathologie-là, donc on a un point en commun. (Inter B)

Cette conception du rétablissement ${ }^{5}$ semble vite assimilée, et elle est expliquée au sociologue dans des discussions informelles. Celles-ci permettent de comprendre pourquoi les usagers auxquels elle a été exposée s'en emparent facilement. Pour l'espoir que cela implique bien sûr, mais aussi comme l'explique l'un d'eux: «Guérir, ça fait peur», «parce que, si on est guéri, alors là, on n'a plus droit à l'erreur». Il y a des bénéfices secondaires à l'idée de rétablissement. Si l'on est rétabli, et non "guéri », on reste fragile et vulnérable, donc on peut revendiquer cette vulnérabilité dans les interactions sociales. En somme, exprimer: je suis seulement rétabli, voire en processus de rétablissement, vous devez prendre des précautions avec moi.

Conception de la personnalité

Les MSP contribuent à diffuser une représentation particulière de la personnalité, de la maladie, de l'expérience et de l'intercompréhension entre êtres humains. Les usagers retiennent des rencontres que seuls peuvent comprendre la maladie et l'état de malade ceux qui les ont connus sous leur étiquette diagnostique, étroite (bipolarité, schizophrénie) ou large (toxicomanie, psychose). Les autres professionnels par leur formation ne peuvent que la «connaître ». Ces représentations-

5. Il s'agit d'une conception parmi d'autres, le mot étant fortement polysémique. Elle connaît d'ailleurs quelques variations chez les MSP eux-mêmes. 
là sont parfois formulées par les MSP, parfois elles émergent de la situation de rencontre et du constat de la présence d'un MSP dans le service.

Dans la même orientation, ne peuvent participer à des groupes d'entendeurs de voix que des entendeurs de voix étiquetés tels, avec le même raisonnement pour les malades «bipolaires» et non des sociologues censés être «normaux». Affirmer qu'un être humain ne peut «comprendre» (par intuition, empathie...) ce qu'il n'a jamais vécu, valoriser le rapport au même, s'oppose à une conception de l'être humain où chacun peut avoir connu au cours de sa vie les mouvements dépressifs, les crises anxieuses, les dérapages, les organisations névrotiques et les noyaux psychotiques, et où toutes les potentialités de la souffrance, de la crise ou du blocage psychique, l'altérité interne, font partie de la vie psychique de chacun d'entre nous.

Le MSP ne remet pas en question les diplômes et la profession des personnels du soin. Mais moi j'ai une différence, c'est que j'ai quelque part des compétences, mais parce que j'ai vécu la maladie. Parce que moi j'ai une vision différente. Vous pouvez très bien avoir un entretien avec une infirmière, vous aurez un entretien, qui sera sans doute intéressant, constructif, mais quand vous aurez un entretien avec moi, il sera différent, parce que moi je n'ai pas la même approche, parce que je suis un ex-patient. (Inter C). MSP

Les infirmières, c'est pas qu'elles nous jugent, c'est que parfois je me demande si elles comprennent. Je me demande si elles comprennent ce par où on est passé. Parce que, elles peut-être, elles ne sont pas passées par là. Elles, elles ont appris, on leur a appris. (U 2)

Parce que quelqu'un qui a fait des études, qui a tous ses diplômes, qui a étudié, qui est sorti avec un diplôme, tout ça, s'il a pas connu la souffrance, il ne peut pas soigner comme quelqu'un qui a souffert. Ce n'est pas possible. (U 45)

L'expérience ne peut que renvoyer à des faits réels et avérés. Elle est ineffable et incommunicable, hors les situations précises où une même expérience (correspondant aux mêmes classifications, nosographiques ou sociales) peut se partager en miroir ${ }^{6}$.

6. Un effet paradoxal est que les MSP, pour soutenir cette conception, sont obligés de faire bouger leurs diagnostics annoncés: soit de les aggraver, soit de les rigidifier, soit encore de les réviser suivant les circonstances. 
Quand elle m'a expliqué qu'elle avait, euh, comme une bête à l'intérieur, là, au début. J'ai dit: "C'est exactement ce que je ressens. » Après elle m'a expliqué qu'au début elle pleurait. Moi aussi. Pendant plusieurs, quelque temps, j'ai pleuré tous les jours. Sans savoir pourquoi, ça venait tout seul. Euh... Enfin, voilà, ses symptômes correspondent bien aux miens, quoi. (U 8)

Critique des autres professionnels et du système de soin

Du fait de leurs rencontres avec les MSP, les usagers se sentent autorisés à formuler certaines critiques vis-à-vis d'autres professionnels ou visà-vis du système de soins. Pourtant les MSP se rendent compte que la critique n'est pas toujours tenable et ils peuvent être capables aussi d'expliquer les contraintes institutionnelles. Il reste que la présence des MSP libère la parole critique. Ces critiques sont souvent nuancées et font des exceptions pour des personnes particulières, n'aboutissant jamais à un dénigrement du groupe professionnel dans son ensemble. Les psychiatres (sauf exception donc) apparaissent plutôt lointains, non souriants, pressés et surtout soucieux de prescriptions médicamenteuses. Les infirmiers pressés, peu disponibles, ne respectant pas les horaires annoncés, tenant des discours normatifs abstraits, voire ne respectant pas l'usager.

Les infirmières c'est tout, elles ont notre dossier, elles connaissent notre parcours parce qu'elles ont notre dossier et puis c'est tout, ça s'arrête là. Tu viens au CATTP c'est bien, tu ne viens pas, c'est pareil, c'est pas grave. Tandis pour le MSP, on n'est pas un dossier, on est une personne [...]. Les infirmières, je les aime bien, mais je vous dis j'ai l'impression par moments qu'on est un dossier. On est voilà, elles ouvrent le dossier: "Madame Untel, qu'est-ce qu'elle a eu comme problème, et qu'est-ce qu'elle a comme genre de troubles? Est-ce qu'elle est bipolaire, est-ce qu'elle est psychotique, schizophrène... ? Le MSP, non. Elle sait qu'on est là parce qu'on a des problèmes, mais avant elle voit une personne. (U 2)

[À propos du psychologue:] J'en ai vu un il y a quelques mois, mais bon il était tout mou, je croyais qu'il était sous médicaments lui-même. (Inter B)

Je n'accroche pas du tout avec la psychiatre, on est jamais d'accord sur rien du tout [...]. C'est sur les médicaments, je trouve toujours que j'en ai trop [...]. Avec les infirmières, elles passent toujours très rapidement sur les critiques envers les médicaments et la critique envers la psychiatre. Elles passent toujours très rapidement là-dessus parce qu'elles savent qu'elles ne peuvent rien faire du tout. Avec le MSP, je peux plus en parler, même si il sait qu'il ne peut rien faire, je peux en parler plus. (U 69) 
En revanche, en cas d'exception et de bonne relation avec un psychiatre ou avec un infirmier, l'utilité différentielle du MSP apparaît beaucoup plus faible, de même si les relations d'entraide entre usagers sont bien développées.

Globalement, les entretiens manifestent la faiblesse de l'offre psychothérapeutique. Les usagers trouvent difficilement l'espace-temps pour verbaliser sur leur passé quand ils en ressentent le besoin: c'est ainsi qu'ils peuvent opposer à la fonction purement médicale des soignants et à la restriction de leur intérêt au temps présent et à sa suite la disponibilité des MSP, pour entendre la parole sur les affects, y compris sur la vie d'avant. Les soignants sont perçus comme ne s'intéressant qu'aux médicaments, à l'humeur présente et aux projets, alors que le MSP plus disponible accepte de discuter de l'affectivité.

Morale de la patience et du petit pas

Sauf exception (en cas par exemple de morale vitaliste valorisant les passions, ou de position plus stoïcienne chez une autre qui se méfie de «l'attente»), la morale de la vie diffusée et celle du prendre soin de soi sont de savoir progresser à "petits pas», autrement dit garder l'espoir en une vie meilleure, mais sans placer la barre des ambitions trop haut. Un MSP: «Il vaut mieux partir sur le moindre et puis quand on a pris ses marques et qu'on se sent bien dans le lieu où on travaille, dire je peux travailler plus.»

U: J'ai des compétences dans certains domaines, mais je n'ai pas les diplômes.

MSP: Regardez-moi, je suis fragile, j'ai une maladie chronique et il y a des moments je vais très mal, j'ai fait le choix de travailler à temps partiel, je travaille $X$ h par semaine. Il y a des soirs où je suis très fatiguée. $U:$ Oui, je veux bien le croire.

MSP: Mais je ne perdrais jamais de vue que il faut que je vois ma santé, d'abord. Parce que si vous mettez la barre trop haut, ce qu'il va arriver, de toute façon ça n'ira pas, vous serez fatigué. (Inter A) MSP

\section{Discussion}

Ce premier article sur les rencontres entre médiateurs de santé pairs et usagers de la psychiatrie a permis de dégager quelques caractéristiques générales de la rencontre: une temporalité peu contrainte, une grande disponibilité relationnelle, une forte proximité. Elles jouent le rôle de révélateur pour les usagers quant à leur vécu de patients et leur 
permettent d'être plus conscients d'un certain nombre d'insatisfactions par rapport aux relations habituelles avec les soignants. Elles leur permettent aussi d'entrevoir un sens de la notion de «rétablissement » et d'espérer ce processus pour eux-mêmes.

En sous-bassement du travail relationnel des MSP, généralement fondé sur le principe de simplicité et d'ouverture des échanges, nous avons aussi identifié une lecture plus normative de la maladie et de l'expérience, qui relève d'une forme de réticences vis-à-vis des savoirs universitaires ou professionnels sur la maladie. Ainsi les MSP peuventils porter une critique relative au système classique de soins, tout en véhiculant par ailleurs un comportementalisme de sens commun, qui se retrouve en accord avec des conceptions scientifiques biomédicales devenues dominantes dans le champ de la santé mentale. Il reste à voir si cet effet sur les représentations des patients se traduit en effets thérapeutiques réels, ce qui sera l'objet du second article consacré aux rencontres des MSP. 